\title{
Epigenetics in Hematological Malignancies
}

\section{Dr. Mostafa El-Razzaz, MD}

\section{Lecturer of Internal Medicine, Hematology and Bone Marrow Transplantation Unit, Ain Shams University}

\section{What is Epigenetics?}

The prefix 'epi' is derived from the Greek preposition 'nope' meaning: 'above'. In biology, 'Epigenetics' is the study of cellular and physiological trait variations that are not caused by changes in the DNAsequence.Broadly, we can consider them semipermanentchanges that effect a set of gene expression changes; however, theyare reversible.

Epigenetic changes are primarily acquired through DNA methylation, which occurs at the cytosine located in a CpG dinucleotide (regions of DNA where a cytosine nucleotide occurs next to a guanine nucleoti

de in the linear sequence of bases along its length), and post-translational histone modifications. These chromatin modifications are usually tightly regulated in development and differentiation. (1)

\section{DNA methylation}

The regulation and maintenance of DNA methylation isessential for appropriate embryonic development, cellular differentiationand genome stability. The catalyticactivity of a family of enzymes known as DNA methyltransferases(DNMTs) results in the addition of a methyl group tothe five-carbon position of cytosine bases in $\mathrm{CpG}$ dinucleotides,yielding 5-methylcytosine $(5 \mathrm{mC})$.DNA methylation has traditionally been thought to mediatetranscriptional silencing and the formation of repressive chromatinstates in addition to maintaining gene expression patternsthrough mitotic cell division. (2)

Disruption of methylation profiles and genome wide loss ofepigenetic stability is observed in malignant transformation. Although aberrant hypermethylation and silencing of tumorsuppressor genes has been found in almost all forms of cancer,both hypomethylation and hypermethylation of promoter $\mathrm{CpG}$ islands can affect the expression of protein coding genesand non-coding RNAs resulting in tumorigenesis.(3) 
Thesechanges are highly disease specific with distinctive methylationpatterns able to distinguish between hematologic malignanciesand even subtypes of these malignancies. (4)

Using emerging high throughput DNA sequencing techniques,recurrent DNMT3A mutations were identified inapproximately $20 \%$ of patients with AML. DNMT3Amutations are enriched in cytogenetically normal, intermediaterisk AML and commonly co-occur with mutations inFms-Related Tyrosine Kinase 3 (FLT3), Nucleophosmin 1(NPM1) and isocitrate dehydrogenase (IDH) $1 / 2$. (5)

DNMT3Amutations have also been identified inpatients with myelodysplastic syndromes (MDS) andmyeloproliferative neoplasms (MPN), and are associatedwith increased likelihood of progression to AML. $(6,7)$

\section{DNA methylation as a therapeutic target in myeloidmalignancies}

Emerging therapeutic strategies targeting epigeneticmechanisms of disease have shown significant promisewith the establishment of DNMT inhibitors as a cornerstoneof management in MDS. DNMT inhibitors such as5-azacitadine and 5-aza-2'-deoxycytidine are nucleosideanalogs that covalently trap DNMT1 following incorporationinto DNA resulting in genome-wide hypomethylationthrough passive dilution of $5 \mathrm{mC}$. (8)

The hypomethylating effects of these agents are at noncytotoxicdose ranges limiting the severity of side effects.Further developmentof the treatment paradigm has suggested that less toxic regimens(lower doses with more frequent dosing) and the useof maintenance DMNT inhibitors as adjunct therapy or incombination with other novel therapies such as lenalidomidemay be effective in subsets of patients with highriskMDS/AML. (9)

\section{DNA hydroxy-methylation and the TET enzymes}

Though DNA methylation was initially believed to be arelatively stable DNA modification, genome-wide high resolutionmapping of $5 \mathrm{mC}$ during cellular differentiation andthe recent identification of the Ten-Eleven-Translocation(TET) enzymes has revealed a more dynamic state ofaffairs. The three TET enzymes (TET1-3) catalyze the successive oxidation of $5 \mathrm{mC}$ to 5 -hydroxymethylcytosine $(5 \mathrm{hmC}), 5$-formylcytosine and 5-carboxycytosine. (10)

The $5 \mathrm{mC}$ derivativeshave beenshown to act as essential intermediates in both active andpassive DNA demethylation, to modulate the binding andrecruitment of chromatin regulators including the polycombrepressive complexes (PRC), and are involved in the reversal of transcriptional silencing. (11)

TET2 hasbeen shown to be mutated in myeloid malignancies includingAML, MDS and MPN with a high proportion of patientswith MDS and chronic myelomonocytic leukemia (CMML)harboring mutations. (12) 
TET2 mutations are enriched inpatients presenting with a normal karyotype, is associatedwith poorer $\mathrm{OS}$ in $\mathrm{AML}$ and $\mathrm{CMML}$ but is not predictiveregarding clinical outcome in MDS and MPN. AlthoughTET2 mutations do not have a strong predictive correlationwith clinical outcome in MDS, TET2 mutations may independentlyact as a biomarker for response to hypomethylatingagents. $(13,14)$

\section{Histone modifications}

The post-translational modification of histone tails by chromatin modifying enzymes has significant impact on intra- and inter-nucleosomal interactions. A considerable number of histone residues can be modified and the diversity of modifications result in highly complex and orchestrated chromatin environments that are dynamically altered in specific cellular contexts. These modifications not onlyhave the ability to regulate the binding of effector moleculesessential to DNA processes including transcription, repairand replication, but also the ability to regulate higher orderchromatin structure and stability.Therefore it is not surprisingthat many chromatin modifying enzymes arederanged during malignant transformation. (15)

Critical protein-protein interactions and essential co-factorsfor enzymatic activity have been identified as viabletherapeutic targets and demonstrate significant promise inthe treatment of malignancies arising from abnormalities inepigenetic regulation. (16)

\section{Acetylation}

Histone acetylation, one of the best studied histone modifications, is dynamically controlled by two opposing familiesof enzymes: lysine acetyltransferases (KATs) and histonedeacetylases (HDACs).KATs are subdivided on the basis of intracellular localizationinto predominantly nuclear (type A) or cytoplasmic (type B)subtypes. Enzymes found in the CBP/p300, MYST andGNAT families are type A KATs. (17)

Recurrent mutations in CBP and p300 are noted in a rangeof hematologic malignancies, especially the lymphoid neo-plasms. Similarly, chromosomal translocations involvingKATs (e.g. MLL-CBPand MOZ-TIF2) are found inmyeloid malignancies. $(18,19,20)$

In general, therapeutic targeting of KATs has thus far beenhampered by their low substrate specificity and broadinvolvement in multi-protein complexes that define theirmolecular activity. Interestingly, a recent structure based insilicoapproach has identified a commercially available, smallmolecule p300/CBP inhibitor; C646. C646 resulted inselective in vitro inhibition of primary human AML bearingthe AML1-ETO translocation through cell cycle arrest andapoptosis. This was associated with a dosedependentreduction in global histone H3 acetylation and decreasedexpression of c-kit and bcl-2. (21)

Recurrent mutations of HDAC's are notobserved in cancer genomes yet HDAC inhibitors havebroadly been trialed in a range of malignancies. This is primarilybecause 
they are aberrantly recruited by variousoncoproteins to inappropriately initiate or maintain malignantgene expression programs. (22)

For instance, the leukemicfusion proteins PML-RARa and PZLF-RAR $\alpha$ have beenshown to recruit HDAC containing repressor complexesresulting in aberrant gene silencing. In murine models ofAPML, the use of HDAC inhibitors (HDACi) is effective inpotentiating or restoring the retinoid-induced differentiation of retinoic acid sensitive and resistant tumors resultingin improved survival. $(22,23)$

The efficacy of HDACi in the treatment of cutaneous Tcelllymphoma has been established. However, the broaderapplication of this class of therapies in other hematologicmalignancies is yet to be clinically proven.Although initially regarded as straightforward activatorsof transcription through direct histone hyperacetylation,a greater appreciation of the non-histone effects ofHDACi on proteins such as p53 and key members of theproteasome/aggresome pathways, HSP90 and tubulin haveemerged. $(24,25)$

Recent mechanistic insight into the antileukemicactivity of HDACi in t(8;21) AML demonstratesthe induction of terminal myeloid differentiation followingHDACi mediated proteasomal degradation of theAML1/ETO9a fusion protein. (26)

Over 40bromodomain containing proteins in eight subfamilies withfunctionally diverse roles such as chromatin remodeling,post-translational histone modificationand transcriptionalco-activation have been identified.For example, highly specific small molecule inhibitors targetingthe protein-protein interactions of the Bromodomainand Extra Terminal (BET) proteins (BRD2, BRD3, BRD4 andBRDt) have emerged as promising therapeutic avenues ininflammation and cancer.Pharmacological BET inhibitionshows remarkable efficacy in vitro and in vivo against MLLfusion leukemia through rapid induction of cell cycle arrestand apoptosis. (27)

Broader extension of pharmacological BET inhibition toother genetically distinct AML subgroups results in theidentification of a core transcriptional program includingcritical oncogenic targets such as BCL2 and C-MYC.

The efficacy of BET inhibition has been replicated in abroad range of hematologic malignancies including multiplemyeloma, non-Hodgkin lymphoma and ALL.These serve as proof of principle for epigenetic targetedtherapies directed against protein-protein interactions, andhave formed the basis for the initiation of early phase clinicaltrials. (28) 


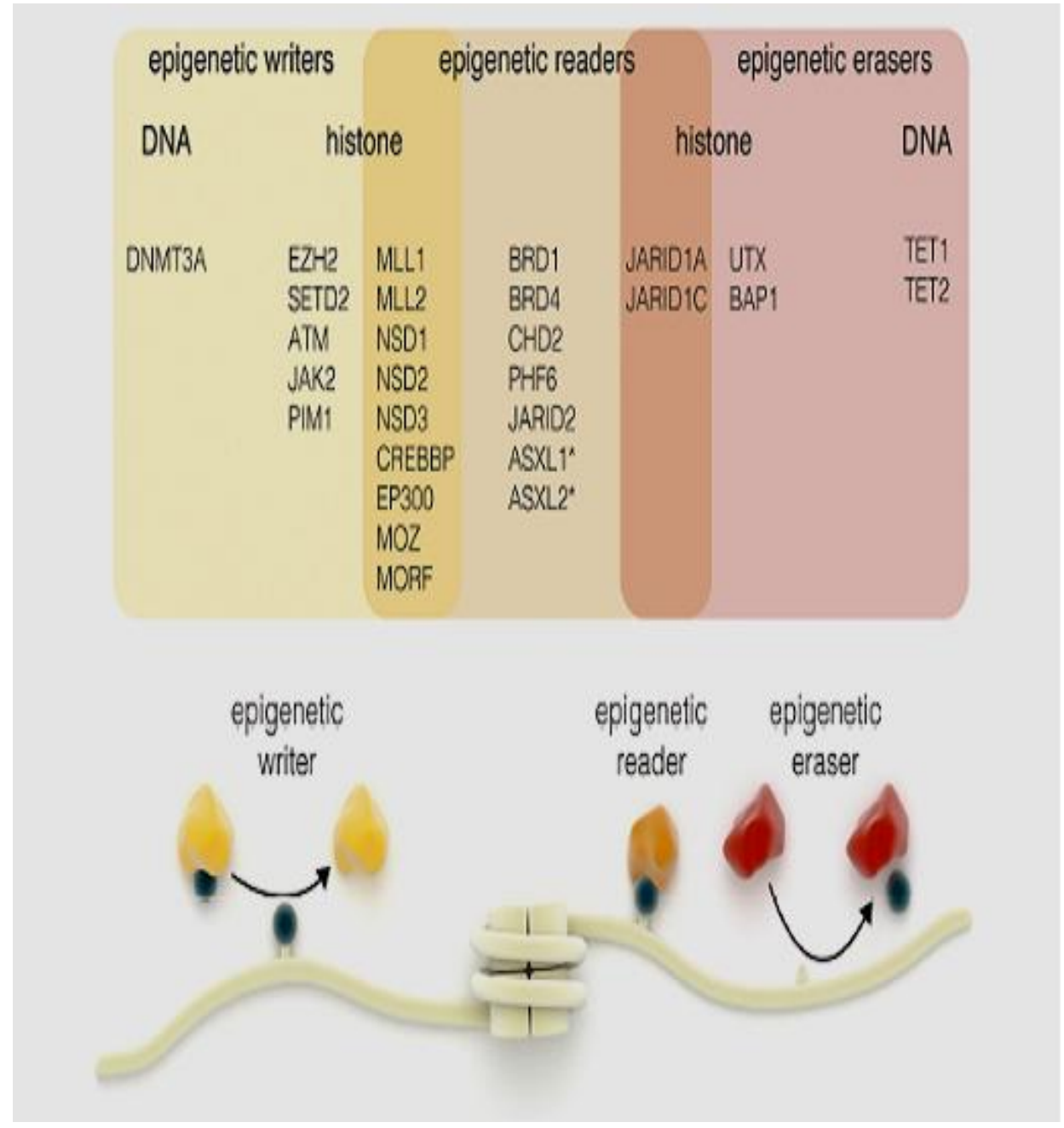

Figure 1:Epigenetic writers, readers and erasers mutated or translocated in hematologic malignancies. Epigenetic writers catalyze the chemical modifications of amino acids on histones or the cytosine base of DNA. Epigenetic erasers catalyze the removal of these modifications and epigenetic readers recognize these modifications and recruit larger macromolecular complexes to the chromatin template. A number of epigenetic writer and erasers also have domains that allow them to function as epigenetic readers (highlighted in the overlap shaded areas).

*ASXL1 and ASXL2 have a PHD domain that may allow them to function as epigenetic readers; however, there is still no conclusive evidence for this. 


\section{Methylation}

Histone methylation occurs predominantly on lysine andarginine residues and is mediated by lysine methyltransferases(KMTs) and protein arginine methyltransferases (PRMTs). (29)

The functional impact of histone methylation is contextualand can lead to both transcriptional activation andrepression.The best-characterized sites of histone lysinemethylation include H3K4, H3K9, H3K27, H3K36, H3K79and H4K20.(30)

Adding to the complexity, themethylation state of individual histone residues also influencesfunctional relevance. For example, monomethylationof H3K9 is associated with active transcription whereastrimethylation is associated with repression116 and, whilstH3K4me2/3 is associated with TSS of active genes,H3K4me1 is associated with active enhancers. (31)

MLL leukemia as a model for therapeutic targetingof disordered epigenetic regulation

Wild-type MLL (WT-MLL) plays an integral role in normalembryogenesis and hematopoiesis. It is a $430 \mathrm{kDaprotein}$ post-translationally cleaved into $\mathrm{N}$-terminal and $\mathrm{C}$ terminalfragments which re-associate to form the MLLcomplex. The C-terminal fragment contains a SETdomain, which methylates H3K4. WT-MLL also has 3HMG-like AT hooks that bind AT rich DNA; a CxxCdomain, four Plant Homeo-Domain (PHD) fingers, a bromodomain,host cell factor binding motif and transactivationdomain mediate interactions with several protein complexes(Figure 2A). (32)

Translocations involving this essential epigenetic regulatoraccount for the vast majority of infantile and approximately $10 \%$ of adult leukemias.MLLleukemias follow anaggressive clinical course with poor response to conventionalchemotherapy and frequent early relapse.(33)

\section{Demethylation}

Analogous to DNA methylation, the discovery ofenzymes capable of reversing lysine methylation has highlightedthe dynamic nature of histone modifications.

In cell line models ofsubtypes of $A M L$, pharmacological inhibition ofKDM1A in combination with ATRA results in reactivationof ATRA-dependent differentiation pathways.Theseeffects were associated with gene-specific, selective increasesin $\mathrm{H} 3 \mathrm{~K} 4 \mathrm{me} 2$ and were respectively associated withdownregulation of genes bound by MLLFP and upregulationof genes associated with myeloid differentiation. 176 

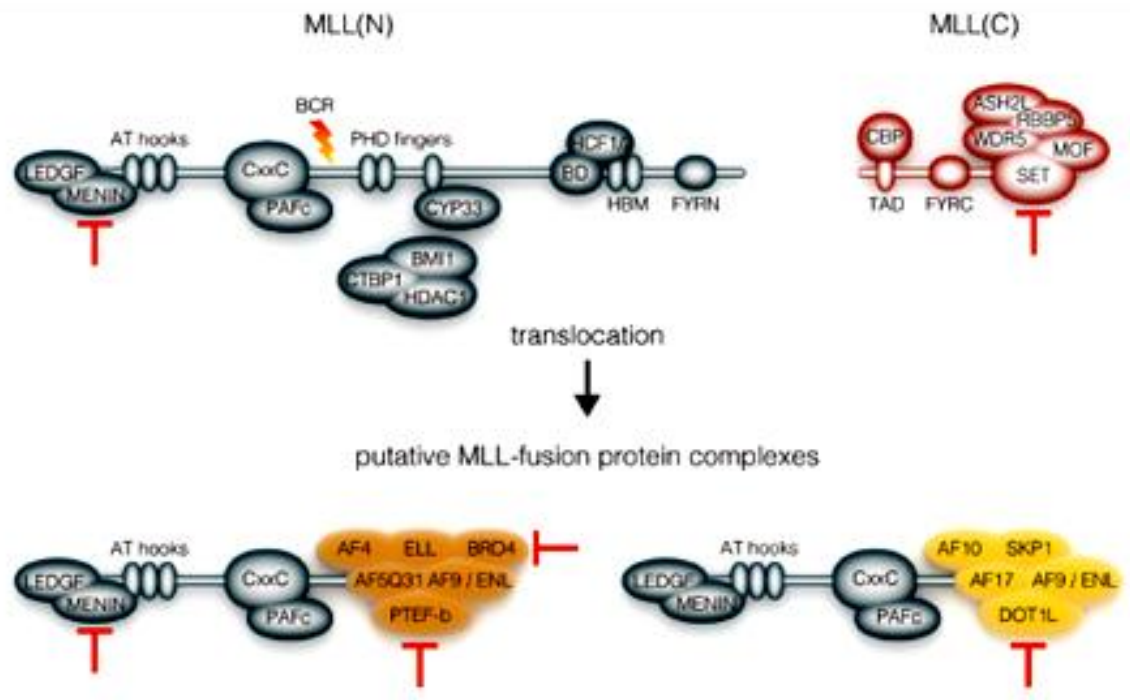

Figure 2:-MLL fusion proteins as targets for small molecule inhibition. Schematic diagram of wild-type MLL illustrating the various specialized domains and the protein-protein interactions mediated by them. Also illustrated are the purported MLL-fusion protein complexes. Following translocation, a fragment of the N-terminal portion of MLL is fused in frame with a translocation partner leading to the formation of novel MLL-fusion protein complexes including the SEC and DOT1L complex. It is unclear whether these are separate entities or co-exist as one large complex. Highlighted are various small molecules that have been developed to target the leukemogenic capacity of either wildtype MLL or MLL-fusion proteins. BCR: breakpoint cluster region; HBM: host cell factor binding motif; TAD: transactivation domain.

\section{Phosphorylation}

Kinases and phosphatases control the addition andremoval of phosphate groups on serine, threonine and tyrosineresidues of component histone proteins. Histone phosphorylation results in gross changes inchromatin structure and has been implicated in the regulationof gene transcription, DNA repair and chromatin condensation.Aberrant kinase activity is one of the most commonlyobserved processes in malignant transformation. (34)

Constitutive activation of JAK2, a non-receptor tyrosinekinase crucial for cytokine signaling in normalhematopoiesis, commonly occurs in MPN. The identification of multiple pathogenic consequencesof aberrant signaling kinase activity at chromatin broadensthe therapeutic scope of kinase inhibitors currently in clinicaldevelopment. Several kinase inhibitors result in globalreduction of histone modification laid down by targetenzymes (e.g. JAK2 and Aurora kinase inhibitors) and thuscan be considered as potential epigenetic therapies. (35) 
Table 1. Current development of targeted epigenetic therapies.

$\begin{array}{ccc}\text { Target enzyme Disease type } & \begin{array}{c}\text { Current stage } \\ \text { of development }\end{array}\end{array}$

\section{Writers}

\begin{tabular}{|c|c|c|c|}
\hline Acetylation & $\begin{array}{l}\text { CBP/p300 A } \\
\text { PCAF }\end{array}$ & $\begin{array}{l}\text { AML, Ovarian, Colon, Melanoma } \\
\text { Ovarian, Colon }\end{array}$ & $\begin{array}{l}\text { Pre-clinical } \\
\text { Pre-clinical }\end{array}$ \\
\hline Methylation & $\begin{array}{l}\text { DOTIL } \\
\text { EZH2 }\end{array}$ & $\begin{array}{c}\text { MLL-r leukaemia } \\
\text { NHL, advanced solid tumors }\end{array}$ & $\begin{array}{l}\text { Clinical } \\
\text { Clinical }\end{array}$ \\
\hline Phosphorylation & $\begin{array}{c}\text { JAK2 } \\
\text { Aurora kinase }\end{array}$ & $\begin{array}{c}\text { MPN } \\
\text { NHL, CML, ALL }\end{array}$ & $\begin{array}{l}\text { FDA approved } \\
\text { Clinical }\end{array}$ \\
\hline \multicolumn{4}{|l|}{ Erasers } \\
\hline Acetylation & HDACI & CTCL & FDA approved \\
\hline Methylation & $\begin{array}{l}\text { LSDI/KDMIA } \\
\text { UTXJMJD3 }\end{array}$ & $\begin{array}{c}\text { AML } \\
\text { Inflammatory response }\end{array}$ & $\begin{array}{l}\text { Clinical } \\
\text { Pre-clinical }\end{array}$ \\
\hline
\end{tabular}

Readers

Acetylation

BET

Haematological malignancies,
NUT midline carcinoma

Clinical

DNA Methylation

DNMT

IDH inhibitors
MDS

AML, glioblastoma
FDA approved

Clinical

MLL-. mixed lineage leukemia rearunged, NHL: nontlodgkin bmphoma; MPN: myeloprobilerotive neoplasms; CML: chronic myeloid leukemia; ALL: acule bmphoblestic leukemia; CTCL: cutaneous Fcell hmphoma; AML: acule myeloid leukemia; MDS: myelodysplastic syndrome.

\section{Conclusions}

Although highly heterogeneousin nature, aberrant regulation of epigenetic processes hasemerged as a prominent unifying theme in hematologicmalignancies.

Somatic alterations of epigenetic regulatorssuch as DNMT3A, TET2,IDH2, MLL, $E Z H 2$ and $A S X L 1$ have prospective prognostic value in AML andMDS.

Therapies directed against epigenetic mechanismsof disease have also entered widespread clinical practicewith resultant improvement in clinical outcomes.

\section{$\underline{\text { References }}$}

1. Shah M, Licht J. (2011):Epigenetics: What Hematologists Need to Know. The Hematologist; 8(3): 14-15.

2. Klose R, Bird A. (2006): Genomic DNA methylation: the mark and its mediators. Trends BiochemSci; 31(2):89-97. 
3. Baylin S, Jones P. (2011):A decade of exploringthe cancer epigenome - biological and translational implications. Nat Rev Cancer; 11(10):726-34.

4. Saied M, Marzec J, Khalid S, et al. (2012):Genome wideanalysis of acute myeloid leukemia revealleukemia specific methylome and subtypespecific hypomethylation of repeats. PloS One; 7(3): 332-13.

5. Ley T, Ding L, Walter M, et al. (2010):DNMT3AMutations in Acute Myeloid Leukemia. N Engl J Med;363(25):2424-33.

6. Walter M, Ding L, Shen D, et al. (2011):Recurrent DNMT3Amutations in patients with myelodysplastic syndromes. Leukemia;25(7):1153-8.

7. Stegelmann F, Bullinger L, Schlenk R, et al. (2011):DNMT3A mutations in myeloproliferative neoplasms. Leukemia;25(7):1217-9.

8. Kantarjian H, Issa J, Rosenfeld C, et al. (2006):Decitabineimproves patient outcomes in myelodysplasticsyndromes: results of a phase III randomized study. Cancer;106(8):1794803.

9. Saunthararajah Y (2013): Key clinical observationsafter 5-azacytidine and decitabine treatmentof myelodysplastic syndromes suggest practicalsolutions for better outcomes. Hematology Am SocHematolEducProgram; 2013:511-21.

10. Tahiliani M, Koh K, Shen Y, et al. (2009): Conversionof 5-methylcytosine to 5hydroxymethylcytosinein mammalian DNA by MLL partner TET1. Science;324(5929):9305.

11. Wu H, Zhang Y. (2011):Tet1 and 5-hydroxymethylation:a genome-wide view in mouseembryonic stem cells. Cell Cycle;10(15):2428-36.

12. Solary E, Bernard O, Tefferi A, et al. (2014): The Ten-ElevenTranslocation-2 (TET2) gene inhematopoiesis and hematopoietic diseases. Leukemia;28(3):485-96.

13. Abdel-Wahab O, Mullally A, Hedvat C, et al. (2009):Genetic characterization of TET1, TET2, and TET3 alterations in myeloid malignancies. Blood;114(1):144-7.

14. Itzykson R, Kosmider O, Cluzeau T, et al. (2011): Impact of TET2 mutations onresponse rate to azacitidine in myelodysplastic syndromes and low blast count acute myeloid leukemias. Leukemia;25(7):1147-52.

15. Kouzarides T. (2007):Chromatin modifications and their function. Cell;128(4):693-705.

16. Dawson M, Prinjha R, Dittmann A, et al. (2011):Inhibition of BET recruitment to chromatinas an effective treatment for MLLfusionleukaemia. Nature;478(7370):529-33.

17. Shahbazian MD, Grunstein M (2007): Functions ofsite-specific histone acetylation and deacetylation. Ann Rev Biochem;76:75-100.

18. Pasqualucci L, Dominguez-Sola D, Chiarenza A, et al. (2011):Inactivating mutations of acetyltransferasegenes in B-cell lymphoma. Nature;471(7337):189-95.

19. Taki T, Sako M, Tsuchida M, et al. (1997):Thet(11;16)(q23;p13) translocation in myelodysplasticsyndrome fuses the MLL gene to the CBP gene. Blood;89(11):3945-50.

20. Carapeti M, Aguiar R, Goldman J, et al. (1998): A novel fusion between MOZand the nuclear receptor coactivator TIF2in acute myeloid leukemia. Blood;91(9):3127-33.

21. Gao X, Lin J, Ning Q, Gao L, et al.(2013): A histone acetyltransferasep300 inhibitor C646 induces cell cycle arrest and apoptosis selectively in AML1-ETO positive AML cells. PloS One;8(2):e55481.

22. Fazi F, Zardo G, Gelmetti V, et al. (2007):Heterochromaticgene repression of the retinoic acid pathwayin acute myeloid leukemia. Blood; 109(10):4432-40.

23. He L, Tolentino T, Grayson P, et al.(2001): Histonedeacetylase inhibitors induce remission intransgenic models of therapy-resistant acutepromyelocytic leukemia. J Clin Invest;108(9):1321-30.

24. Bolden J, Peart M, Johnstone R. (2006):Anticancer activities of histone deacetylaseinhibitors. Nat Rev Drug Discov;5(9):769-84.

25. Dickinson M, Johnstone R, Prince H. (2010):Histone deacetylase inhibitors: potential targetsresponsible for their anti-cancer effect.Invest New Drugs;28 (Suppl 1):S3-20. 
26. Bots $M$, Verbrugge I, Martin B, et al. (2014):Differentiation therapyfor the treatment of $t(8 ; 21)$ acutemyeloid leukemia using histone deacetylase inhibitors. Blood;123(9):134152.

27. Filippakopoulos P, Qi J, Picaud S, et al. (2010):Selective inhibition of BET bromodomains. Nature;468(7327):1067-73.

28. Mertz JA, Conery A, Bryant B, et al. (2011):Targeting MYC dependence in cancer byinhibiting BET bromodomains. Proc NatIAcadSci USA;108(40):16669-74.

29. Allis C, Berger SL, Cote J, et al.(2007): Newnomenclature for chromatin-modifying enzymes. Cell;131(4):633-6.

30. Barski A, Cuddapah S, Cui K, et al. (2007):High-resolutionprofiling of histone methylations in the human genome. Cell;129(4):823-37.

31. Heintzman N, Stuart R, Hon G, et al. (2007):Distinctand predictive chromatin signatures oftranscriptional promoters and enhancers inthe human genome. Nat Genet;39(3):311-8.

32. Bernt K, Armstrong S. (2011):Targeting epigeneticprograms in MLL-rearrangedleukemias. Hematology Am SocHematolEduc Program;2011:354-60.

33. De Braekeleer M, Morel F, Le Bris M, et al. (2005): The MLL gene andtranslocations involving chromosomal band11q23 in acute leukemia. Anti cancer Res;25(3B):1931-44.

34. Hanahan D, Weinberg R. (2011):Hallmarks ofcancer: the next generation. Cell;144(5):646-74.

35. Liu F, Zhao X, Perna F, et al. (2011):JAK2V617F-mediatedphosphorylation of PRMT5 downregulatesits methyltransferase activity and promotesmyeloprolife-ration. Cancer Cell;19(2):283-94.

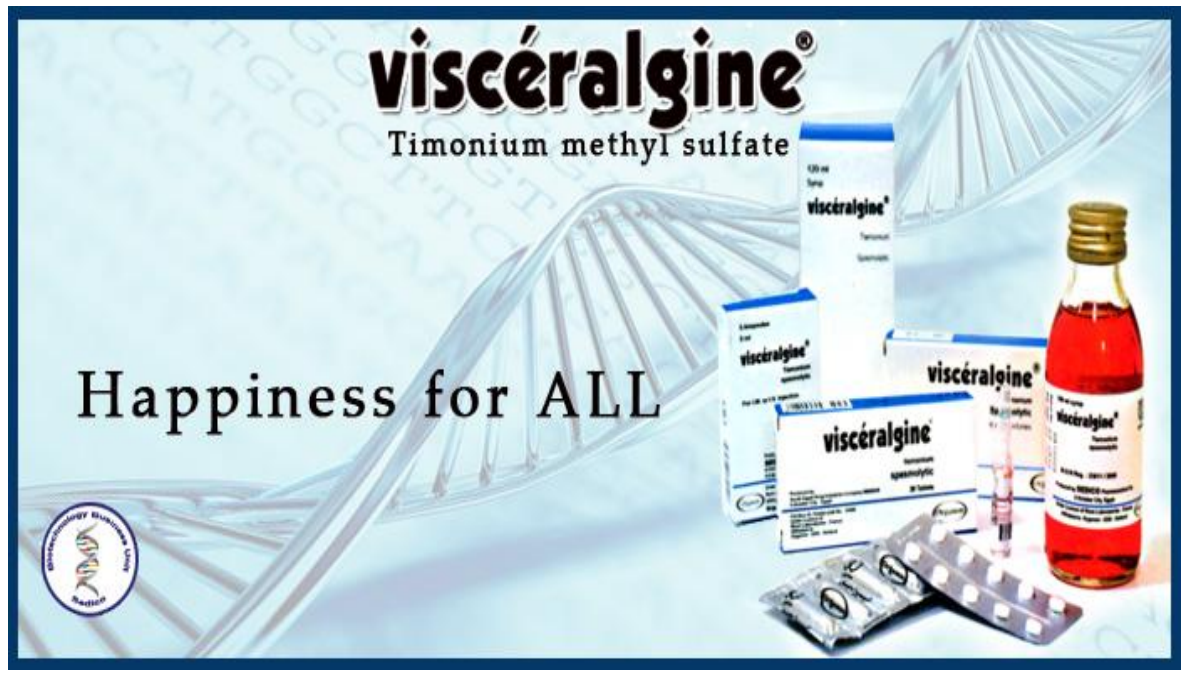

